\title{
Myocarditis Post mRNA COVID-19 Vaccine- To Fear or Not to Fear?
}

\author{
Adeeba Ahmed ${ }^{1 *}$, Anam Lodhi ${ }^{2}$ and Mishal Shan Siddiqui ${ }^{1}$ \\ ${ }^{1}$ Dow Medical College, Karachi, Pakistan \\ ${ }^{2}$ Karachi Medical and Dental College, Karachi, Pakistan
}

\section{Dear Editor,}

We consider effective mass vaccination as the sole way to curb the COVID-19 pandemic. This is made even more pertinent by the virus's ever-mutating nature and increased transmissibility. Most side effects of the vaccine are minor and self-limiting such as injectionsite pain, swelling, fever, and muscle aches. However, a more concerning side effect, myocarditis, is also emerging.

Myocarditis is inflammation of the heart's muscular layer [1], which presents with symptoms ranging from chest pain to arrhythmias, even shock. Julia et al. reported 1226 cases of post-vaccine myocarditis in which males aged 16 years or above were most vulnerable and developed symptoms following the second dose of the mRNA vaccine [1]. The mechanism of pathogenesis, however, is not elucidated. A hypothesis suggests a higher antibody response to the vaccine while another attributes it to a cytokine storm caused by some antiidiotype cross-reactive antibody in the myocardium [2].

Amid the prevailing myths, reports about such side effects add to the public's fear of vaccination [3]. This subsequently undermines the efforts to eradicate COVID-19. Thus, we believe it is important to highlight that the side effect is rare and manageable without any significant long-term damage. As per a prediction, for every 1 million individuals, we could prevent 11000 COVID-19 infections, 560 hospitalisations, 138 ICU admissions and 6 deaths by vaccination alone [1]. This is opposed to $39-47$ cases of myocarditis, thus, clearly favouring vaccination [1]. The risk of myocarditis with COVID-19 infection is much greater than with the vaccine [1]. One should also emphasise that the overall vaccine effectiveness is $94.1 \%$ [4] and thus, the larger population can safely ignore this side effect.

In situations where the risk of myocarditis is anticipated, preventive measures can be implemented. Individuals at risk including adolescent males, those with a previous history of myocarditis or other adverse cardiovascular events should be identified and addressed accordingly. Advising the at-risk subjects to avoid strenuous activity post-vaccination might also be beneficial [5]. Individuals should be informed about the symptoms and guided to contact their doctor if needed. Myocarditis should be actively investigated if chest pain with fever and dyspnoea occurs, especially after the second dose of vaccine [1]. Patients can be managed with supportive measures, NSAIDs, or steroids without any permanent heart damage [5].

However, to devise population-specific guidelines, we recommend that the incidence of post-vaccine myocarditis is actively reported and published in our country, and measures are taken accordingly.

\section{CONFLICT OF INTEREST}

The authors declare no conflict of interest.

\section{ACKNOWLEDGEMENTS}

None.

\section{REFERENCES}

1. Gargano JW, Wallace M, Hadler SC, Langley G, Su JR, Oster ME, et al. Use of mRNA COVID-19 vaccine after reports of myocarditis among vaccine recipients: update from the Advisory Committee on Immunization Practices-United States, June 2021. MMWR Morb Mortal Wkly Rep 2021; 70(27): 977-82.

2. Das BB, Moskowitz WB, Taylor MB, Palmer A. Myocarditis and pericarditis following mRNA COVID-19 vaccination: What do we know so far? Children (Basel) 2021; 8(7): 607.

3. Solís Arce JS, Warren SS, Meriggi NF, Scacco A, McMurry N, Voors $\mathrm{M}$, et al. COVID-19 vaccine acceptance and hesitancy in low-and middle-income countries. Nat Med 2021; 27(8): 1385-94.

4. Information about the Moderna COVID-19 Vaccine. Centers for Disease Control and Prevention. Available at: https://www.cdc. gov/coronavirus/2019-ncov/vaccines/different-vaccines/Moderna. html (Accessed on: October 09, 2021)

5. Bozkurt B, Kamat I, Hotez PJ. Myocarditis with COVID-19 mRNA Vaccines. Circulation 2021; 144(6): 471-84.

\footnotetext{
*Corresponding author: Adeeba Ahmed, Dow Medical College, Karachi, Pakistan; Email: adeebaahmed098765@gmail.com 